\title{
Is an equal interval scale an equal discriminability scale?**
}

\author{
HENRY MONTGOMERY† \\ University of Göteborg, Göteborg, Sweden \\ and \\ HANNES EISLER \\ University of Stockholm, Stockholm, Sweden
}

\begin{abstract}
Stevens and Galanter's (1957) iterative procedure for minimizing bias in category scaling was used for the scaling of loudness of white noise. The spacing obtained deviated systematically from a spacing constructed in accordance with an equal discriminability scale from a previous experiment (Eisler \& Montgomery, 1972). For the stimulus spacing yielding a "pure" category scale, a magnitude scale was constructed too. Since the category scale could be predicted accurately by Fechnerian integration of this magnitude scale, it was concluded that the "pure" category scale is a pure discrimination scale. The discrepancy between the equal discriminability scale and the "pure" category scale was interpreted as a bias in the former scale due to greater recognizability of stimuli located at the extremes of the stimulus range.
\end{abstract}

Several experiments have demonstrated that the form of a psychophysical scale can be affected by the particular stimuli selected (Garner, 1954; Pradhan \& Hoffman, 1963; Stevens, 1958; Stevens \& Galanter, 1957). This seems to be particularly true for rating scales or category scales (Stevens, 1958). A rationale for stimulus spacing effects on category ratings has been given by Stevens and Galanter (1957), who assumed that when doing category rating the $S$ expects to employ the category numbers approximately equally often. This assumption was also made by Parducci in his range frequency model (Parducci, 1965). Parducci's model has been shown to predict a large proportion of those changes in category scale values that result from varying the relative frequency and spacing of stimulus values (Parducci, 1965; Parducci \& Perret, 1967, 1971).

The bias that may result from a typical S's expectations of being required to use the category numbers equally often could perhaps be counteracted by a suitable monotone transformation of the responses (cf. Anderson, 1962, 1972). An alternative method would be to select the stimuli in a way which meets these expectations as nearly as possible. Stevens and Galanter suggested that this could be achieved by using an iterative procedure which aims at producing category scale values with equal intervals between successive stimuli.

This procedure was used by Pollack (1965) on category ratings of the brightness of gray papers. Pollack tested the procedure on several different sets of gray

\footnotetext{
*This paper was supported by a grant from the Swedish Council for Social Science Research. The authors want to thank J. Liljencrants for his technical assistance and the Department of Speech Transmission, Royal Institute of Technology, Stockholm, for making their facilities a vailable.

tRequests for reprints should be sent to Henry Montgomery. Department of Psychology, University of Göteborg, Box 14094, S-400 20 Göteborg 14, Sweden.
}

papers and found that the iterative procedure yielded a single empirical scaling from the different sets of stimuli. It is worth noting that Pollack's scales converged on a predetermined spacing of category scale values. The possibility cannot be excluded that the scales would also converge on another spacing of category scale values if the iterative procedure were to be based on that particular spacing. It should also be noted that Pollack did not report any direct test of the assumption that Ss tend to use the category numbers equally of ten when they rate the set of stimuli selected by the iterative procedure. One of the aims of the experiment reported below is to investigate the validity of this assumption.

The basic aim of the procedure outlined above is apparently to neutralize bias in the category scale values. In a recent paper by the authors (Eisler \& Montgomery, 1972), the problem of selecting appropriate stimuli for category ratings was taken up from another angle. In earlier papers, the second author had demonstrated that the category scale can be predicted by Fechnerian integration of the magnitude scale (Eisler, 1962, $1963 \mathrm{~b}, \mathrm{c})$. Fechnerian integration, as we use the term, corresponds to a special case of the General Psychophysical Differential Equation (the GPDE) (Eisler, 1963a, 1965; Eisler, Holm, \& Montgomery, 1973) which expresses the following relation

$$
\frac{\mathrm{dy}}{\mathrm{dx}}=\frac{\sigma_{\mathrm{y}}(\mathrm{y})}{\sigma_{\mathrm{x}}(\mathrm{x})}
$$

where $\mathrm{x}$ and $\mathrm{y}$ are two subjective variables and $\sigma_{\mathrm{y}}(\mathrm{y})$ and $\sigma_{\mathbf{x}}(\mathrm{x})$ their Weber functions (usually defined as the intraindividual SDs as a function of the central tendencies). As can be seen from (1), one subjective variable, say $y$, can be predicted from the other when both Weber functions are known. This procedure 
coincides with Fechnerian integration when the Weber function of the $y$ variable is constant. (For the case at hand, $y$ denotes the category scale and $x$ the magnitude scale). In the earlier tests of the Fechnerian model, the Weber function of the category scale was assumed to be constant. However, the empirically obtained SDs were greatest in the middle of the stimulus range and decreased toward both ends. One way of dealing with this discrepancy between empirical data and theory is to regard the Weber function of the category scale as subject to distortion or bias. In the Eisler and Montgomery study (1972), it was suggested that the smailer SDs for the more extreme stimuli can be seen as an "end effect" due to greater recognizability of stimuli located at the ends of the stimulus range (Garner, 1952). This bias can be minimized, however, by selecting a spacing of stimuli in such a way that the identifiability of the stimuli will be as equal as possible. This was achieved by constructing an equal discriminability (ED) scale in accordance with Garner and Hake (1951).

Thus, two criteria for the selection of stimuli in category ratings have been proposed: (1) to choose the stimuli so that the category scale intervals between successive stimuli are equal (Stevens \& Galanter, 1957), and (2) to select the stimuli so that the identifiability of the stimuli is as equal as possible (Eisler $\&$ Montgomery, 1972).

The latter approach aims at minimizing bias in the Weber function of the category scale, whereas the former has as its objective to neutralize bias in the category scale values themselves. It seems reasonable to assume that the two criteria are closely related. Stated more precisely, if the category scale is a discriminability scale, as indicated by the Fechnerian model, it may be expected that the equal discriminability (ED) scale should be linearly related to the category scale, i.e., equal discriminable steps between successive stimuli (ED spacing) should correspond to equal intervals on the category scale. However, the ED scale being computed from responses in an absolute judgment task has proved to be biased for the extreme stimuli due to the greater recognizability of these stimuli in absolute judgment tasks (Garner, 1952). It was noted above that also in category rating the extreme stimuli may be easier to recognize than the remaining stimuli. However, if we assume that a greater recognizability of the extreme stimuli in category rating only affects the Weber function but not the category scale values, a nonlinear relation between the category scale and the ED scale should be expected. In fact, the relation between the two scales should be sigmoid, since a greater recognizability of the extreme stimuli implies that the slope of the ED scale will be steeper at the extremes of the stimulus continuum. This hypothesis was confirmed in the Eisler and Montgomery study (1972), indicating that the category scale could be a less biased discriminability scale than the ED scale.

The above considerations suggest that it may be impossible to neutralize bias in both the category scale and its Weber function simultaneously by using the same spacing of stimuli. However, if the Fechnerian model is used for predicting the category scale, one might expect the accuracy of the model to be maximal if the bias in the category scale is kept at a minimum.

The major objective of the present investigation was to study the category scale and its Weber function for the ED spacing and for a spacing of stimuli selected by the procedure described by Stevens and Galanter (1957). The continuum of white noise was chosen for the study.

The following predictions were tested: (1) The number of judgments assigned to each category will be approximately the same for the spacing of stimuli selected using Stevens and Galanter's technique. (2) The ED spacing will be systematically different from the spacing of stimuli selected using Stevens and Galanter's technique. (3) The category scale of the stimuli selected using Stevens and Galanter's technique can be predicted by Fechnerian integration of a magnitude scale for the same spacing of stimuli.

\section{METHOD}

\section{Design and Subjects}

An iterative category rating experiment, following the principles described by Stevens and Galanter (1957), was conducted for loudness of white noise. All in all, six successive iterations were carried out (see Table 1). The initial spacing of stimuli was identical to the approximate ED spacing employed in the Eisler and Montgomery study (1972). As can be seen in Table 1, the loudest noise in that spacing was $110 \mathrm{~dB}$, which may seem quite loud. It is possible that the effective sound level of this noise was lower than the physical calibration indicated, due to the relative ineffectiveness of lower frequencies for very high sound levels. However, this is of no consequence, since in the present case it would be quite sufficient to have stimulus measures on the ordinal level to be able to test the predictions stated above. Different Ss were used for each iteration, and the number of Ss used for each set of stimuli is also presented in Table 1.

A magnitude estimation experiment was carried out with the spacing of stimuli that came closest to the criterion of the iterative procedure (Spacing 6 in Table 1). Twenty Ss participated in this experiment.

All the Ss were undergraduate students of psychology at the University of Stockholm.

Ten stimuli (intensities of white noise) were used in all experiments.

\section{Apparatus}

A keyboard was connected to a computer (CDS 1700) which both monitored the presentation of the stimuli and recorded the responses. The keyboard was covered by a template with holes for those buttons that were actually being used by the Ss. Ten of these buttons were assigned an integer from either 1 to 10 (in the category rating experiment) or from 0 to 9 (in the magnitude estimation experiment), and in the magnitude estimation experiment, there was also a button corresponding to the decimal point. There were two more buttons, one marked "next noise" and the other "erase."

The white noise was produced in a noise generator and then passed via an amplifier adjusted to an SPL of $110 \mathrm{~dB}$ (re 0.00002 microbar), an attenuator (Fonema, smallest step $.5 \mathrm{~dB}$ ), a bandpass filter (manufactured by the Swedish Telecommunications Administration, bandwidth 75-2,400 cps, 
Table 1

Stimulus Spacings in dB (S), Category Scale Values (C), and Number of Subjects in the Iterative Category Rating Experiment

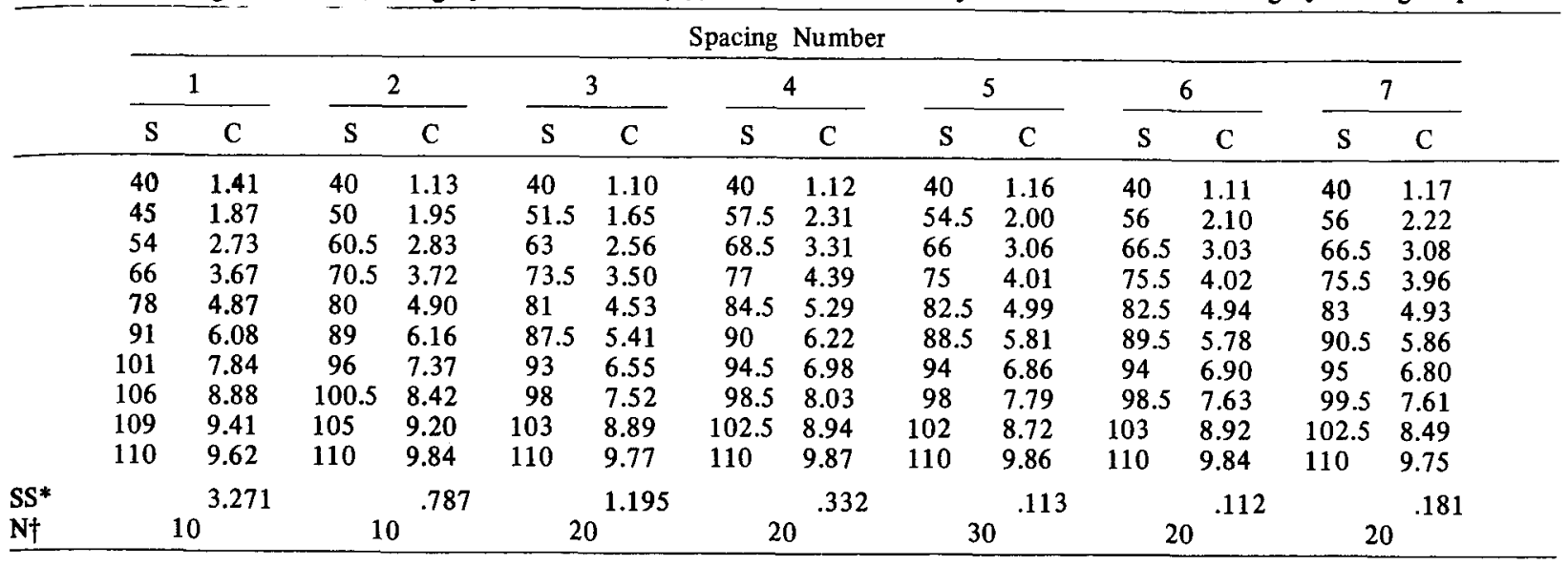

* Sums of squared deviations between category scale values and equal intervals between successive stimuli. tNumber of $S$ s.

slope of filter function $12 \mathrm{~dB} /$ octave), and a matching transformer in to a pair of earphones (Beyer T48, 5 $\Omega$ ). The onset and offset of each stimulus was practically instantaneous. The intensity of the stimuli was controlled by the computer via the attenuator.

In the magnitude estimation experiment, the computer was connected to an oscilloscope which displayed to the $S$ his response on each trial.

\section{Procedure}

In the category rating experiment, the $S$ was presented with the weakest and strongest intensities $(40$ and $110 \mathrm{~dB}$ in all the iterations) and informed that they were called 1 and 10 , respectively. The $\mathrm{S}$ was instructed to assign to each stimulus noise an integer between 1 and 10 so that successive subjective sensation intervals between successive numbers were equal. A 10-point category scale was used in order to have the same number of categories and stimuli. This was to avoid skewed distributions of responses when the category scale intervals between successive stimuli were approximately equal.

In the magnitude estimation experiment, a stimulus of medium intensity $(82.5 \mathrm{~dB})$ was presented to the $S$ and called 10 (the standard). The $\mathrm{S}$ was then asked to estimate the loudness of the stimulus noises so that the ratio between the numbers given and 10 reflected the ratio between the sensations of the stimuli presented and the standard.

The subsequent procedure was the same for both of the experiments.

Following the presentation of the standatd or the standards there were 10 preliminary trials, one for each of the 10 different stimulus intensities. A random presentation order was used. The experiment proper started with the re-presentation of the standard or the standards. After this there were 100 trials consisting of 10 blocks within which each stimulus was presented once. The computer selected completely new random orders for each block and for each $S$.

In all the experimental conditions each stimulus was initiated immediately as the button marked "next noise" was pressed, and it lasted until the next time this button was pressed, i.e., the presentation persisted during the period when the judgment button(s) was (were) pressed. If the $S$ wanted to correct his just given judgment, he could press the button marked "erase" instead of "next noise," make his new judgment, which replaced the previous one, and proceed by pressing the button "next noise." The $S$ was allowed to wait as long as he wished before pressing any of the buttons. The rationale is that Ss will listen to a noise for the time necessary to make a judgment. Presenting the noises for fixed duration might satisfy the E's sense of order, but would most probably not equate listening times subjectively.

\section{Selection of Stimulus Values in the Iterative Experiment}

The stimulus values in the iterative category rating experiment were selected in accordance with the procedure described by Pollack (1965), which is as follows: (1) Plot the initial category scaling as a function of the decibel value of the stimuli. (2) Determine the difference between the mean category ratings assigned to the upper and lower stimulus limits. (3) Divide the difference by $(n-1)$, where $n$ is the number of stimuli used for the iterative scaling (10 in the present experiment). (4) Add the quotient of Step 3 successively to the mean category rating assigned to the lower limit until the rating assigned to the upper limit is reached. (5) Draw a line parallel to the abscissa from each of the values of Step 4 to the smoothed curve. (6) Drop a perpendicular from the intersection to the abscissa. (7) Read the stimulus values on the abscissa scale.

As a measure of the goodness of an iteration, we used the sum of the squared deviations between the empirical category scale and the category scale predicted by the iterative procedure (see Table 1). The iterations were stopped when the sum of the squared deviations seemed to have reached the state of random fluctuations.

\section{RESULTS}

\section{Category and Magnitude Scales}

Category scale values were computed by taking the arithmetic mean of the estimations gained for each stimulus, over all trials and Ss. The magnitude scale values were calculated by taking the arithmetic mean of the 10 judgments made by each $S$ for each stimulus and the geometric mean of the arithmetic means of all the Ss. This method corresponds to a method for computing intraindividual standard deviations (Eisler, 1962) which was used on the results to be reported below (cf. "Weber Functions").

It can be seen in Table 1 that the sum of the squared deviations between empirical and predicted category scales was reasonably stable after three iterations. The fit is best for Spacing 6 though practically the same for Spacing 5 . Table 1 also shows that the category scales 


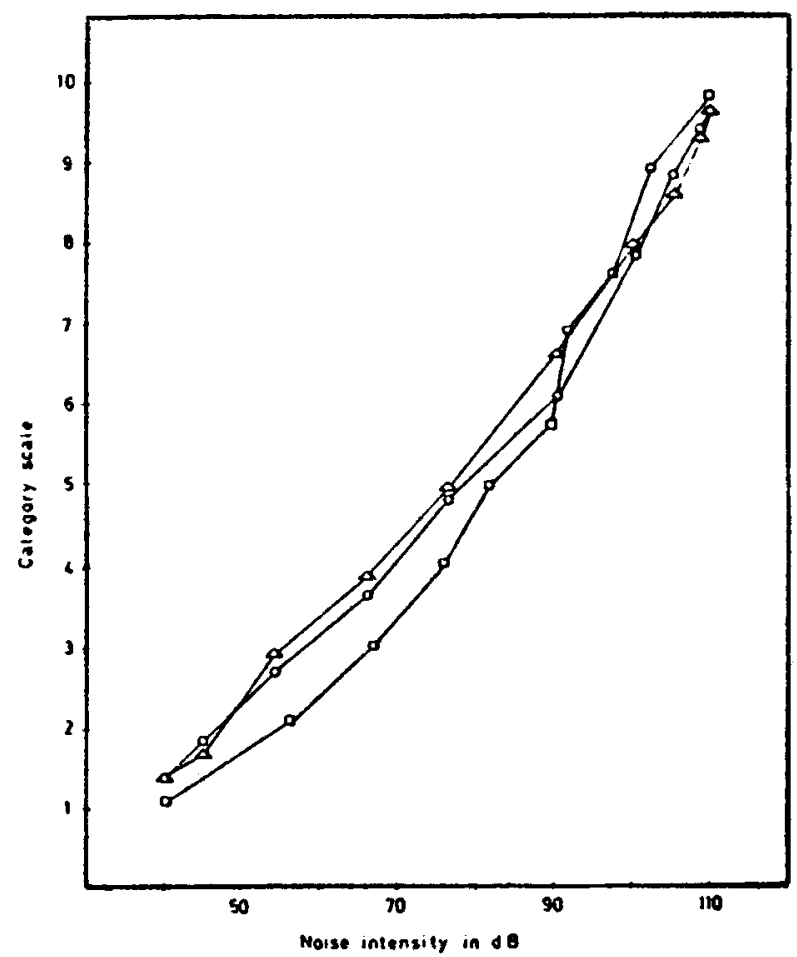

Fig. 1. Category scales for loudness as a function of noise intensity [circles: Spacing 1; squares: Spacing 6; triangles: a category scale from an experiment reported by Eisler and Montgomery (1972). The latter scale was subjected to a linear transformation to make it correspond to the category scales from Spacings 1 and 6.]

Table 2

Percentage of Assignments for Each Category for the Category Scales from Spacings 5 and 6

\begin{tabular}{ccrrrc}
\hline \multirow{5}{*}{$\begin{array}{c}\text { Spacing } \\
\text { Number }\end{array}$} & 1 & 2 & 3 & 4 & 5 \\
\hline \cline { 2 - 6 } 5 & 12.1 & 10.3 & 9.1 & 11.3 & 8.7 \\
6 & 12.3 & 9.1 & 10.9 & 10.3 & 9.7 \\
\cline { 2 - 6 } & 6 & 7 & 8 & 9 & 10 \\
\cline { 2 - 6 } 5 & 8.3 & 8.6 & 10.8 & 10.3 & 10.5 \\
6 & 9.0 & 7.9 & 9.5 & 9.9 & 11.5 \\
\hline
\end{tabular}

for Spacings 5 and 6 are very close to a scale with equal intervals between successive stimuli. The final spacings deviate conspicuously from the initial spacing (see Table 1) which was made identical to the ED spacing used in the Eisler and Montgomery study (1972). The latter spacing becomes more crowded the more extreme a stimulus is, whereas the former spacings become more crowded the greater the stimulus value, with an exception for the two loudest stimulus noises.

In Fig. 1 the category scales of Spacings 1 and 6 are plotted in semilog coordinates against stimulus values. Both scales are concave upward. However, the trend is more marked for the category scale of Spacing 6 , with an exception for the upper limit of this scale which exhibits a slight reversal. Figure 1 also shows the category scale of the ED spacing in the Eisler and Montgomery study (1972). It can be seen that the agreement is rather good between this scale and the category scale of Spacing 1 despite the differences in experimental procedure.

Table 2 gives the percentage of judgments assigned to each category of the category scales of Spacings 5 and 6 . For both spacings, the number of judgments is relatively high for the two extreme categories, 1 and 10, and relatively low for the innermost categories, 5, 6, and 7 . A Friedman test for matched groups indicated that the effect of stimulus number on the number of judgments assigned to each category was statistically significant for the two spacings referred to in Table $2\left(\chi^{2}=24.55\right.$, $\mathrm{p}<.005$ for Spacing 5 and $\chi^{2}=23.12, \mathrm{p}<.01$ for Spacing 6).

Figure 2 shows the category scale for Spacing 6 as a function of the magnitude scale for the same spacing. As can be seen, the function relating the category scale to the magnitude scale is concave downward.

\section{Weber Functions}

In Fig. 3 intraindividual SDs of the category ratings are plotted against the category scale values from each of the spacings of stimuli. The SDs were calculated by averaging the individual variances. The curves in Fig. 3 are parabolas $\left(\sigma=k_{K}(K-a)(b-K)\right.$, where $\sigma=$ intraindividual $\mathrm{SD}, \mathrm{K}=$ category scale values, and $\mathrm{k}_{\mathrm{K}}, \mathrm{a}$, and $b$ are constants). The parabolas were fitted by the method of least squares. It can be seen that the fit of the parabola is good for all the spacings, and may even be

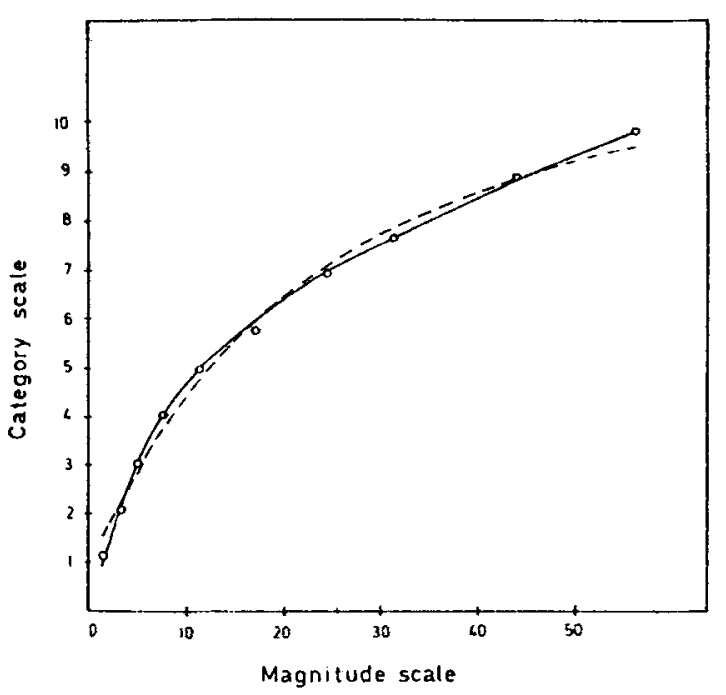

Fig. 2. The category scale for Spacing 6 as a function of the magnitude scale for the same spacing. The curves constitute predictions of the category scale allowed by the GPDE for two combinations of Weber functions (continuous curve: parabolic-constant combination; dashed curve: parabolic-parabolic combination). 

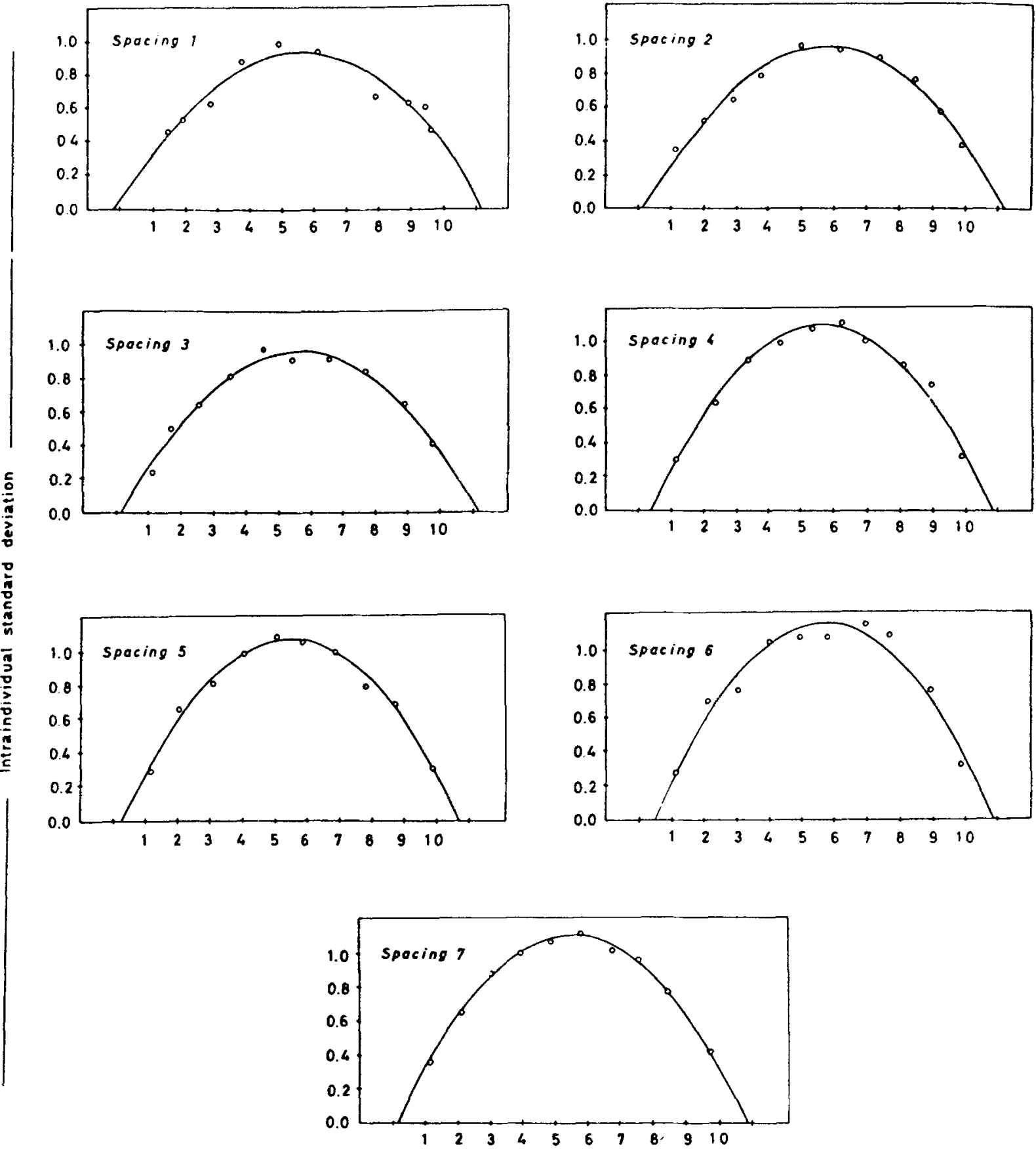

Category scale

Fig. 3. Intraindividual SDs as a function of category scale values of loudness for Spacings 1-7. The curves are parabolas which were fitted by the method of least squares.

regarded as excellent for some of them. The parameters of the fitted parabolas are given in Table 3. In all parabolas the left K-intercept ( $a$ in the equation of the parabola) is somewhat lower than the lowest category number and the right $\mathrm{K}$-intercept $(\mathrm{b}$ in the equation of the parabola) somewhat higher than the highest category number.

Figure 4 shows intraindividual SDs of the magnitude estimations as a function of the magnitude scale values. The SDs were calculated in accordance with a method 
Table 3

Parameters of Parabolas $\sigma=\mathbf{k}_{\mathbf{K}}(\mathbf{K}-\mathbf{a})(\mathbf{b}-\mathbf{K})$ Fitted to Intraindividual SDs as a Function of Category Scale Values

\begin{tabular}{cccc}
\hline $\begin{array}{c}\text { Spacing } \\
\text { Number }\end{array}$ & $\mathrm{k}_{\mathrm{K}}$ & $\mathrm{a}$ & $\mathrm{b}$ \\
\hline 1 & .0284 & -.14 & 11.30 \\
2 & .0311 & .14 & 11.21 \\
3 & .0318 & .18 & 11.16 \\
4 & .0401 & .40 & 10.86 \\
5 & .0398 & .28 & 10.67 \\
6 & .0428 & .47 & 10.87 \\
7 & .0383 & .14 & 10.89 \\
\hline
\end{tabular}

described by Eisler (1962). The Weber function in Fig. 4 was fitted by a parabola according to another method also described by Eisler (1962). The fit of the parabola can be regarded as good. The equation of the fitted parabola is $\sigma=0.00328(\psi+1.04)(112.77-\psi)$. As can be seen, the left $\psi$-intercept is rather close to zero and the right $\psi$-intercept is about twice as great as the largest scale value.

\section{Fechnerian Integration and the GPDE}

As mentioned in the introduction, earlier studies have demonstrated that the category scale can be regarded as a Fechner integral of the magnitude scale. Fechnerian integration corresponds to a special case of the GPDE, viz, the case with one of the Weber functions constant. We now assume that the genotypic, i.e., unbiased, Weber function of the category scale is constant and that the phenotypic, i.e., empirically obtained, Weber function of the category scale is parabolic. In the following data treatment, the genotypic and phenotypic Weber functions of the category scale are compared. It is assumed throughout that the Weber function of the magnitude scale is parabolic. Inserting the genotypic Weber function into the GPDE as well as the parabolic Weber function of the magnitude scale yields

$$
\frac{\mathrm{dK}}{\mathrm{d} \psi}=\frac{\mathrm{k}}{\mathrm{k}_{\psi}\left(\psi-\mathrm{a}_{\psi}\right)\left(\mathrm{b}_{\psi}-\psi\right)}
$$

where $\mathrm{K}$ and $\psi$ denote the category and magnitude scale, respectively, and $\mathrm{k}$ the constant Weber function of the category scale. Integrating Eq. 2 yields

$$
\mathrm{K}=\frac{\mathrm{k}}{\mathrm{k}_{\psi}\left(\mathrm{b}_{\psi}-\mathrm{a}_{\psi}\right)} \ln \frac{\psi-\mathrm{a}_{\psi}}{\mathrm{b}_{\psi}-\psi}+\mathrm{C}
$$

Inserting the phenotypic Weber function of the category scale into Eq. 2 yields

$$
\frac{\mathrm{dK}}{\mathrm{d} \psi}=\frac{\mathrm{k}_{\mathrm{K}}\left(\mathrm{K}-\mathrm{a}_{\mathbf{K}}\right)\left(\mathrm{b}_{\mathrm{K}}-\mathrm{K}\right)}{\mathrm{k}_{\psi}\left(\psi-\mathrm{a}_{\psi}\right)\left(\mathrm{b}_{\psi}-\psi\right)}
$$

Integrating Eq. 4 yields

$$
K=\frac{a_{K}+b_{K} \cdot C\left(\frac{\psi-a_{\psi}}{b_{\psi}-\psi}\right)^{\frac{k_{K}\left(b_{K}-a_{K}\right)}{k_{\psi}\left(b_{\psi}-a_{\psi}\right)}}}{1+C\left(\frac{\psi-a_{\psi}}{b_{\psi}-\psi}\right)^{\frac{k_{K}\left(b_{K}-a_{K}\right)}{k_{\psi}\left(b_{\psi}-a_{\psi}\right)}}} .
$$

The GPDE was tested by Eqs. 3 and 5. The integration constant $\mathrm{C}$ as well as the constants $\mathrm{k} / \mathrm{k}_{\psi}$ and $\mathrm{k}_{\mathrm{K}} / \mathrm{k}_{\psi}$ were fitted by minimizing the sum of squares $\Sigma\left(K^{\prime}-K\right)^{2}$, where $K^{\prime}$ denotes predicted and $K$ empirical category scale values. Chandler's program (1969) was used. The expressions $\mathrm{k} / \mathrm{k}_{\psi}$ and $\mathrm{k}_{\mathrm{K}} / \mathrm{k}_{\psi}$ can be regarded as measures of the ratio between the absolute levels of the Weber functions. The reason for treating these expressions as free parameters was that the absolute sizes of the uncertainties in category rating and magnitude estimation, respectively, do not seem to be quite comparable in terms of the GPDE (Eisler, 1963a; Eisler \& Montgomery, 1972; Eisler, Holm, \& Montgomery, 1973).

Figure 2 demonstrates that the fit of the category scale predicted by Fechnerian integration (Eq. 3) is almost perfect. The stress ${ }^{1}$ defined as $\sqrt{\Sigma(\mathrm{K}}-$ $\left.\mathrm{K}^{\prime}\right)^{2} / \Sigma \mathrm{K}^{2}$ is .011 . The category scale predicted by Eq. 5 (phenotypic Weber function of the category scale) deviates slightly, but systematically from the empirical category scale values as shown by Fig. 2. The stress is .037. The values of the constants $\mathrm{k} / \mathrm{k}_{\psi}$ and $\mathrm{k}_{\mathrm{K}} / \mathrm{k}_{\psi}$, when fitted as described above, are 264.04 and 11.63 , respectively. The corresponding values of these expressions, when computed from the parameters of the fitted Weber functions, are 252.13 and 13.05 (k was

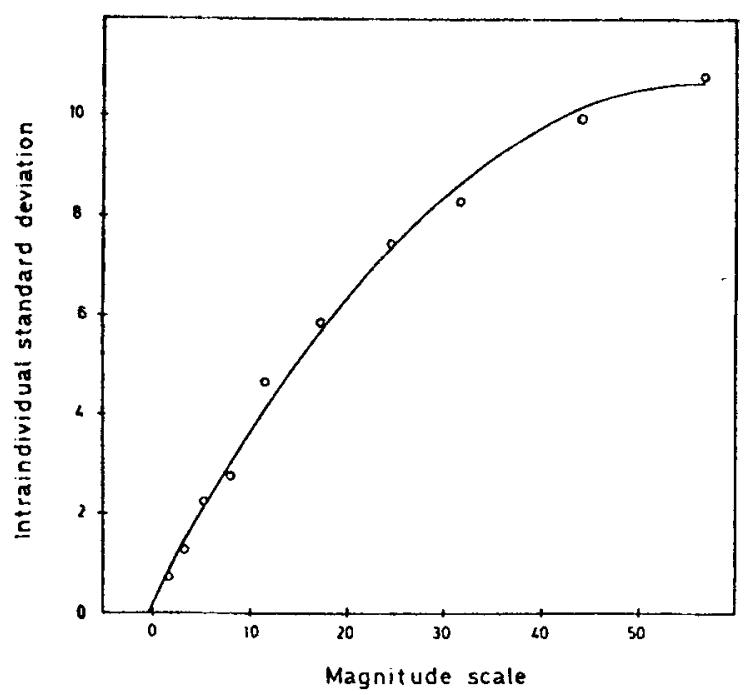

Fig. 4. Intraindividual SDs as a function of magnitude scale values of loudness for Spacing 6 . The curve is a parabola which was fitted by a variation of the method of least squares (Eisler, 1962). 
computed as the square root of the mean variance of the category ratings for each stimulus). Consequently, the ratio between the absolute levels of the Weber functions was rather well estimated by the GPDE in the present experiment, especially by Eq. 3 (Fechnerian integration).

\section{DISCUSSION}

The iterative category rating experiment resulted in a category scale which came close to the criterion of the iterative procedure, i.e., a scale with equal intervals between successive stimuli. However, the prediction that the number of assignments to each category would be the same did not prove completely true. As Table 2 shows, the two extreme categories, 1 and 10 , were used somewhat more often than expected. This is not surprising, however, since the SDs were very low for the two extreme stimuli and markedly higher for the adjacent stimuli, implying that the two extreme categories were used almost always for the two extreme stimuli, whereas the judgments of the adjacent stimuli are distributed over more than one category. Thus, the relatively high frequencies of assignments to Categories 1 and 10 need not be explained in terms of response bias. However, it seems difficult to justify the same conclusion regarding the relatively low frequencies of assignments for the innermost categories $(5,6$, and 7$)$. These results may reflect a tendency to avoid the innermost categories, an observation that gets support from Stevens and Galanter's (1957) report from a 7-point category rating experiment which clearly indicated that the Ss tended to avoid Category 4.

It should be pointed out, however, that the deviations from a constant frequency of assignments to each category were rather small. Thus, this category scale is close to a pure category scale in the sense of Stevens and Galanter. The accurate predictions yielded by Fechnerian integration may also indicate that this category scale was relatively unbiased. It can be noted that the fit of the Fechnerian model as measured by the stress value was approximately twice as good as in the Eisler and Montgomery study (1972).

The close fit of the Fechnerian model again supports the hypothesis that the category scale is a discrimination scale. However, it should be noted that the measure of discrimination in the Fechnerian model is computed from responses in a magnitude estimation task, whereas, for example, Garner and Hake's ED scale is computed from responses in an absolute judgment task. This implies that agreement between a Fechnerian discrimination scale, as defined by Eisler, and the ED scale is only to be expected when equal degrees of discriminability in the Fechner integral of magnitude estimations correspond to equal degrees of discriminability in absolute judgments. The discrepancies between the ED spacing and the final spacing of stimuli in the iterative experiment indicate in fact that the ED scale does not agree with a Fechnerian discrimination scale.

It was noted above that the ED scale has proved to be biased at the ends of the scale due to the greater recognizability of the extreme stimuli in the absolute judgment task. If a corresponding bias existed in the Fechnerian discrimination scale, one might expect that the SDs for the extreme stimuli in a magnitude estimation task would be too small compared to the SDs of the other stimuli. However, this seems not to be the case in the present magnitude estimation experiment (cf. also Eisler \& Montgomery, 1972; Montgomery, 1971). Thus, a Fechnerian discrimination scale could be a more unbiased discriminability scale than the ED scale. An experiment reported by Eriksen and Hake (1957) indicated that the greater recognizability of the extreme stimuli in an absolute judgment task is associated with the fact that the ends of the response continuum are specified in the instruction given in an absolute judgment task. This implies that the extreme stimuli in magnitude estimation may not be more easily recognized than the remaining stimuli, since the magnitude estimation instruction does not usually specify any end responses to be used by the S (cf. Montgomery, 1971).

The discrepancies between the ED spacing and the final spacings of stimuli in the present investigation confirm the introductory hypothesis that it is impossible to neutralize bias in both the category scale and its Weber function with the same spacing of stimuli. It should be pointed out, however, that the Weber function of the category scale for the initial spacing (the ED spacing) was clearly parabolic, whereas the Weber function of the 7-point category scale for the corresponding spacing in the earlier Eisler and Montgomery study was approximately constant with the exception of the weakest stimulus and the two strongest stimuli. The reason for this discrepancy could be that the present study employed 10-point category scales. In other words, the discrepancy between the two Weber functions may indicate that only category scales with a relatively small number of categories can exhibit approximately constant phenotypic Weber functions. This hypothesis is supported by the fact that an approximately parabolic Weber function was obtained for a 15-point category scale of the ED spacing in the Eisler and Montgomery study (1972).

The parabolic Weber functions that were obtained for both the category scales and the magnitude scale are in line with previous findings (Montgomery, 1971). In the case of category scales, the intercepts of the parabola were located symmetrically around the end points of the response scale. This inverted $U$ trend could be explained in terms of adaptation level theory. If this theory is qualitatively correct, then the loudest noise, which is always preceded by weaker noise, will always be perceived as relatively loud. The corresponding reasoning holds for the weakest noise, and to a diminishing degree as the positions of the noises recede from the end points. 
On the other hand, the middlemost noise (or noises) will fluctuate upwards or downwards in loudness according to the particular preceding noises. Hence, perceived loudness will show more variability in the center of the stimulus range. ${ }^{2}$ However, when using this line of reasoning it is not obvious why the Weber function should be parabolic. Eisler (1960) has suggested that a parabolic Weber function may indicate that the $S$ is working with two anchorage points and that his uncertainty is proportional to the distance from each of the anchorage points. In a subsequent paper, we will develop this idea as to its generality and its implications for the relationship between different types of direct scales.

\section{REFERENCES}

Anderson, N, H, On the quantification of Miller's conflict theory. Psychological Review, 1962, 69, 400-414.

Anderson, N. H. Algebraic models in perception. Technical Report No. 30, November 1972, Center for Human Information Processing, University of California, San Diego.

Chandler, J. P. STEPIT-Finds local minima of a smooth function of several parameters. Behavioral Science, 1969, 14, 81-82.

Eisler, $H$. Similarity in the continuum of heaviness with some methodological and theoretical considerations. Scandinavian Journal of Psychology, 1960, 1, 69-81.

Eisler, H. Empirical test of a model relating magnitude and category scales. Scandinavian Journal of Psychology, 1962, 4, $88-96$.

Eisler, H. A general differential equation in psychophysics: Derivation and empirical test. Scandinavian Journal of Psychology, 1963a, 4, 265-272.

Eisler, H. How prothetic is the continuum of smell? Scandinavian Journal of Psychology, 1963b, 4, 29-32.

Eisler, H. Magnitude scales, category scales, and Fechnerian integration. Psychological Review, 1963c, 70, 243-253.

Eisler, H. On psychophysics in general and the general psychophysical differential equation in particular. Scandinavian Journal of Psychology, 1965, 6, 85-102.

Eisler, H., Holm, S., \& Montgomery, H. Is the general psychophysical differential equation an approximation? Reports from the Psychological Laboratories, University of Stockholm, 1973, No. 386.

Eisler, H., \& Montgomery, H. On theoretical and realizable ideal conditions in psychophysics: Magnitude and category scales and their relation. Göteborg Psychological Reports, 1972, 2, No, 16.

Eriksen, C. W., \& Hake, H. W. Anchor effects in absolute judgments. Journal of Experimental Psychology, 1957, 53, 132-138.

Garner, W. R. An equal discriminability scale for loudness judgments. Journal of Experimental Psychology, 1952, 43, 232-238.

Garner, W. R. Context effects and the validity of loudness scales. Journal of Experimental Psychology, 1954, 48, 218-224.

Garner, W. R., \& Hake, H. W. The amount of information in absolute judgments. Psychological Review, 1951, 58, 446-459. Montgomery, H. Direct estimation: Effects of methodological factors on scale type. Göteborg Psychological Reports, 1971, 1 , No. 9.

Parducei, A. Category judgment: A range frequency model. Psychological Review, 1965, 72, 407-418.

Parducci, A., \& Perret, L. R. Contextual effects for category judgments by practiced subjects. Psychonomic Science, 1967, 9, 357-358.

Parducci, A., \& Perret, L. F. Category rating scales: Effects of relative spacing and frequency of stimulus values. Journal of Experimental Psychology, 1971, 89,427-452.

Pollack, I. Neutralization of stimulus bias in the rating of grays. Journal of Experimental Psychology, 1965, 69, 564-578.

Pradhan, P. L., \& Hoffman, P. J. Effect of spacing and range of stimuli on magnitude estimation judgments. Journal of Experimental Psychology, 1963, 66, 533-541.

Stevens, J. C. Stimulus spacing and the judgment of loudness. Journal of Experimental Psychology, 1958, 56, 246-250.

Stevens, S. S $m$ \& Galanter, E. H. Ratio scales and category scales for a dozen perceptual continua. Journal of Experimental Psychology, 1957, 51, 377-411.

\section{NOTES}

1. Stress was used as a measure of deviation to obtain comparability between different experiments.

2. The authors are indebted to the reviewer of this paper for offering this explanation of the form of the Weber functions.

(Received for publication August 17, 1973; revision received December 1,1973 .) 